\title{
Atrioventricular nodal reentrant tachycardia ablation with radiofrequency energy during ongoing tachycardia: is it feasible?
}

\author{
Basri Amasyali ${ }^{1}$, Ayhan Kilic $^{2}$, Kutsi Kabul ${ }^{2}$, Murat Unlu² \\ ${ }^{1}$ Department of Cardiology, School of Medicine, Dumlupinar University, Kutahya, Turkey \\ ${ }^{2}$ Department of Cardiology, Gulhane Military Medical School, Ankara, Turkey
}

Postep Kardiol Inter 2014; 10, 4 (38): 301-307

DOI: $10.5114 /$ pwki.2014.46775

\begin{abstract}
A bstract
Introduction: Radiofrequency (RF) ablation of the slow pathway for treatment of atrioventricular nodal reentrant tachycardia (AVNRT) is conventionally performed during sinus rhythm.

Aim: To evaluate the clinical and electrophysiological features and the short- and long-term results of slow pathway RF ablation during ongoing AVNRT.

Material and methods: A total of 282 consecutive patients with AVNRT undergoing RF catheter ablation were analysed. Patients whose tachycardia episodes could not be controlled during RF energy application and who underwent slow pathway ablation or modification during ongoing tachycardia formed the study group (group $1, n=16$ ) and those ablated during sinus rhythm formed the control group (group 2, $n=266$ ).

Results: Of the clinical characteristics, only the frequency of tachycardia attacks was higher in group $1(3.3 \pm 1.2$ vs. $2.1 \pm 0.9$ attacks/month, $p<0.001)$. Among the baseline electrophysiological measurements, the echo zone lasted significantly longer in group 1 than in group 2 ( $78 \pm 25$ ms vs. $47 \pm 18 \mathrm{~ms} ; p<0.001$ ). The immediate procedural success rate was $100 \%$ in both groups. There were no significant differences between groups regarding the mean number of radiofrequency energy applications $(5.2 \pm 4.2$ vs. $5.8 \pm 3.9)$, total procedure times $(42.4 \pm 30.5 \mathrm{~min}$ vs. $40.2 \pm 29.4 \mathrm{~min})$ and fluoroscopy times $(11.4 \pm 8.5 \mathrm{~min}$ vs. $12.2 \pm 9.3 \mathrm{~min})$ $(p>0.050$ for all). All patients were followed-up for $29 \pm 7$ months; only 2 patients $(<1 \%)$ in group 2 recurred $(p>0.050)$. No permanent atrioventricular block was observed.

Conclusions: The RF catheter ablation or modification of the slow pathway during ongoing AVNRT is feasible with acceptable short- and long-term efficacy and safety. However, this approach needs to be clarified with large-scale studies.
\end{abstract}

Key words: atrioventricular nodal reentrant tachycardia, catheter ablation, supraventricular tachycardia, slow pathway ablation.

\section{Introduction}

Atrioventricular nodal reentrant tachycardia (AVNRT) is the most common form of paroxysmal regular supraventricular tachycardia in adults and accounts for $60 \%$ of these tachycardias [1]. Slow pathway radiofrequency (RF) ablation is considered the first-line therapy for AVNRT. The usual approach is conventional catheter ablation or modification of the slow pathway during sinus rhythm. However, episodes of AVNRT may be incessant in its clinical presentation, and it may be difficult to control the tachycardia and maintain sinus rhythm during electrophysiological (EP) study [2-4]. Not infrequently, recurrent episodes of AVNRT are encountered during RF energy application, due either to a spontaneous extrasystole or the triggering effect of the energy applied to the target site, which is frustrating for both the patient and the staff.

\begin{abstract}
Aim
The aim of this study was to report the clinical and EP characteristics of patients who have undergone slow pathway ablation or modification during ongoing tachycardia as well as the short- and long-term safety and efficacy of this approach in a large series of consecutive patients with AVNRT.
\end{abstract}




\section{Material and methods}

\section{Patients}

We reviewed the records of 282 consecutive patients treated with RF catheter ablation for haemodynamically tolerable symptomatic AVNRT. All patients had a history of paroxysmal palpitations ranging from 5 months to 45 years. The estimated frequency of tachycardia episodes ranged from once every 6 months to 7-8 attacks per month. Those patients whose tachycardia episodes could not be controlled during RF application and who underwent slow pathway ablation or modification during ongoing tachycardia formed the study group (group 1, $n=16)$. The decision to continue slow pathway ablation or modification during ongoing tachycardia was made in patients in whom AVNRT was still induced by junctional beats or atrial premature contractions occurring during RF energy application after three such attempts. Patients who underwent formal ablation during sinus rhythm formed the control group (group 2, $n=266$ ). Patients proven to have an additional accessory pathway or another form of arrhythmia other than AVNRT were excluded. The study was approved by the institutional ethical committee, and informed written consent was obtained from each patient for the procedure.

All patients underwent 12-lead surface ECG, X-ray examination, echocardiography, and blood testing including thyroid hormones and, when clinically indicated, stress testing and 24-hour rhythm Holter recording.

\section{Electrophysiological study and radiofrequency catheter ablation}

The EP study and catheter ablation were performed in a single session in all patients in the fasting, unsedated state and after discontinuation of all antiarrhythmic drugs for at least five half-lives. Written informed consent for the EP study and ablation was obtained from each patient.

The standard protocol consisted of decremental high right atrial $\left(A_{1} A_{1}\right)$ pacing, usually starting from 500 ms and decreasing in steps of $10 \mathrm{~ms}$ until the atrioventricular (AV) node Wenckebach cycle length was reached, and single atrial extrastimulus $\left(A_{1} A_{2}\right)$ testing at three different drive train cycle lengths $(600,500$, and $450 \mathrm{~ms})$ to induce tachycardia. During pacing with the first drive train, the $A_{1}-A_{2}$ interval was shortened by 10 ms until the $A V$ node effective refractory period (ERP) had been reached. A jump of the $\mathrm{AH}$ interval was defined as the difference between any consecutive $\mathrm{AH}$ intervals $\geq 50 \mathrm{~ms}$ during programmed or incremental atrial pacing. The ventriculoatrial (VA) interval of the tachycardia was recorded as the duration between the beginnings of the surface QRS and the A wave recorded from the high right atrial catheter.

All EP data were collected with the patients unsedated and before infusion of any pharmacological stimulants. If the tachycardia was not inducible, 1-2 mg atropine was administered intravenously and the same protocol was repeated. The AVNRT was diagnosed according to standard criteria $[5,6]$.

Slow pathway ablation was done using $7 \mathrm{Fr}$ quadripolar deflectable catheters with 4-mm tip electrodes (Marinr MC, Medtronic Co, Minneapolis, MN). All patients underwent ablation with electrogram-guided anatomic approach. Fifty watts of energy with a temperature limit of $65^{\circ} \mathrm{C}$ was applied at successful sites for $60 \mathrm{~s}$ to $90 \mathrm{~s}$. RF delivery was terminated if junctional rhythm did not appear in the first $15 \mathrm{~s}$ in those patients undergoing ablation during sinus rhythm. In patients who were undergoing ablation during ongoing tachycardia, RF delivery was applied for $30 \mathrm{~s}$ to $45 \mathrm{~s}$ to compensate for possible delayed heating of the catheter tip caused by tachycardia. If tachycardia was still continuing after 45 s, RF energy was terminated to look for a new target site. If tachycardia stopped during the first 30-45 s of energy application, then RF energy delivery to the same target site was continued until $90 \mathrm{~s}$, unless catheter dislodgement occurred with termination of the tachycardia. In case of catheter dislodgement, the catheter was repositioned to the same point and RF application continued to complete $90 \mathrm{~s}$ of energy delivery.

Special attention was paid to patients undergoing ablation during ongoing tachycardia. As the atrial electrogram waves are not readily discernible in most episodes of typical AVNRT, sites with large ventricular (V) electrograms were targeted, as these were thought to be associated with smaller atrial (A) waves (A-to-V electrogram ratio of less than 0.5 ). This was achieved by positioning the catheter at the posterior and sometimes middle third of the triangle of Koch, anterior to the coronary sinus, slightly superior to the tricuspid annulus insertion into the inferior interatrial septum. The coronary sinus ostium was considered an important landmark, and ablation was performed under $40^{\circ}$ left anterior oblique projection after confirmation of the final catheter tip position in the right anterior oblique projection.

The endpoints of ablation were demonstration of either slow pathway block or slow pathway modification with no more than one single echo beat along with noninducibility of AVNRT, both at basal condition and after infusion of 1-2 mg atropine, unless sinus tachycardia was present, after a waiting period of 30 min following ablation.

\section{Follow-up after radiofrequency catheter ablation}

All patients were discharged within $24 \mathrm{~h}$ postoperatively. After hospital discharge, all patients were scheduled for a visit after 4-6 weeks and every 3 months thereafter in the first year. If palpitations recurred, patients were asked to obtain an ECG as soon as possible and contact our centre. 


\section{Statistical analysis}

All continuous data were expressed as mean \pm standard deviation or median (range). Groups were compared by means of $\chi^{2}$ analysis or Fischer's exact test when needed for discrete variables and with unpaired Student's $t$ test for continuous variables. The paired $t$ test was used to compare the continuous data before and after ablation.

Statistical comparisons were performed using the statistical software package SPSS 10.01 (SPSS Inc., Chicago, IL). Calculated $p$ values of less than 0.05 were considered significant in all statistical tests.

\section{Results}

\section{Clinical characteristics}

Among baseline clinical characteristics, only the frequency of the tachycardia attacks was significantly higher in group 1 as compared to patients in group 2 (3.3 \pm 1.2 vs. $2.1 \pm 0.9$ attacks/month, $p<0.001$ ). There was a predominance of hypertensive cases (5 (31\%) vs. 60 $(23 \%))$, structural heart diseases (9 (56\%) vs. 92 (35\%)) and a tendency toward older age ( $48 \pm 17$ years (range: 19-84) vs. $41 \pm 16$ years (range: $17-78)$ ) in group 1 , which were not statistically significant ( $p>0.050$ for all) (Table I).

\section{Electrophysiological characteristics}

The EP characteristics of patients are shown in detail in Table II. Discontinuous AV nodal conduction curves were found in 218 (77\%) patients, distributed similarly in groups 1 and 2 (75\% vs. 77\%, $p>0.050)$. Antegrade AV node ERP, Wenckebach cycle length and tachycardia cycle length were slightly longer in group 1 than in group 2, which were not statistically significant $(p>0.050$, for both). Among the EP characteristics, only the echo zone was seen to last significantly longer in group 1 than in group 2 (78 \pm 25 ms vs. $47 \pm 185 \mathrm{~ms}, p<0.001)$.

\section{Slow pathway ablation or modification}

The results of RF ablation are shown in Table III. The AVNRT was eliminated with right endocardial approach in all cases. Of the 218 patients with discontinuous AV nodal function curves, the antegrade jump disappeared in 170 patients (78\%) and persisted with or without single AV nodal echo beats in 48 patients (22\%) after ablation. Of the 48 patients with residual dual pathway physiology, 3 cases (19\%) were in group 1 and 45 cases $(17 \%)$ in group 2 , not reaching statistical significance $(p>0.050)$. Fluoroscopy $(12.2 \pm 9.3 \mathrm{~min}$ vs. 11.4 $\pm 8.5 \mathrm{~min}$ ) and procedure durations (42.4 $\pm 30.5 \mathrm{~min}$ vs. $40.2 \pm 29.4 \mathrm{~min}$ ) were slightly longer and the number of RF pulses was slightly higher $(5.8 \pm 3.9$ vs. $5.2 \pm 4.2)$ in group 1 , which were not statistically significant ( $p>0.050$ for all). However, in order to avoid bias, RF energy applications made before the decision to proceed with ablation during ongoing tachycardia were not included in the statistical analysis. No major complications were seen in either group. Transient second-degree Mobitz type I AV block lasting less than $30 \mathrm{~s}$ was seen in 1 patient in group $2(<1 \%)$.

To check for residual inducible tachycardia, atropine infusion was done $30 \mathrm{~min}$ after the ablation in 14 patients (88\%) in group 1 and in 225 patients (85\%) in group 2 , which showed no statistically significant difference $(p>0.050)$.

Permanent PR lengthening was observed in 2 patients $(<1 \%)$ in group 2 and none in group $1(p>0.050)$.

Table I. Baseline clinical characteristics of groups

\begin{tabular}{lccc} 
Parameter & Group $1(n=16)$ & Group 2 $(n=266)$ & Value of $p$ \\
\hline Age, mean \pm SD (range) [years] & $48 \pm 17(19-84)$ & $41 \pm 16(17-78)$ & 0.066 \\
\hline Male/female & $44 / 56$ & $46 / 54$ & NS \\
\hline Systemic hypertension, $n(\%)$ & $5(31)$ & $60(23)$ & NS \\
\hline Diabetes mellitus, $n(\%)$ & $4(25)$ & $45(17)$ & NS \\
\hline Structural heart disease, $n(\%):$ & $9(56)$ & $92(35)$ & 0.079 \\
\hline Valvular heart disease & $4(25)$ & $42(16)$ & NS \\
\hline Coronary artery disease & $3(19)$ & $40(15)$ & NS \\
\hline Left ventricular hypertrophy & $3(19)$ & $31(12)$ & NS \\
\hline LVEF $<50 \%$ & $1(6)$ & $19(7)$ & NS \\
\hline Duration of symptoms, mean \pm SD [years] & $11.5 \pm 6.7$ & $176(66)$ & NS \\
\hline Medication, $n$ (\%): & $12(75)$ & $64(24)$ & NS \\
\hline$\beta$-Blockers & $4(25)$ & $83(31)$ & NS \\
\hline Calcium channel blockers & $5(31)$ & $29(11)$ & NS \\
\hline Other antiarrhythmic agents & $3(19)$ & $2.1 \pm 0.9$ & $<.001$
\end{tabular}

LVEF-left ventricular ejection fraction, SD - standard deviation, NS - not significant 
Table II. Electrophysiological characteristics of groups

\begin{tabular}{lccc} 
Parameter & Group $1(n=16)$ & Group 2 $(n=266)$ & Value of $p$ \\
\hline AV node WCL, mean \pm SD [ms] & $330 \pm 23$ & $325 \pm 32$ & NS \\
\hline AV node antegrade ERP, mean \pm SD [ms]: & $236 \pm 10$ & $231 \pm 22$ & NS \\
\hline AV node FP- ERP* & $323 \pm 33$ & $313 \pm 25$ & NS \\
\hline AV node SP-ERP* & $237 \pm 12$ & $232 \pm 22$ & NS \\
\hline Maximum AH interval, mean \pm SD [ms] & $324 \pm 36$ & $318 \pm 35$ & NS \\
\hline AH jump, $n(\%)$ & $12(75)$ & $206(77)$ & NS \\
\hline Multiple AH jumps, $n(\%)$ & $4(25)$ & $33(12)$ & NS \\
\hline Echo zone, mean \pm SD (range) $[\mathrm{ms}]$ & $78 \pm 25(40-130)$ & $47 \pm 185(20-110)$ & NS \\
\hline Type of AVNRT, $n(\%):$ & & & NS \\
\hline Typical & $14(88)$ & $248(93)$ & NS \\
\hline Atypical & $2(13)$ & $337 \pm 40$ & NS \\
\hline TCL, mean \pm SD [ms] & $356 \pm 44$ & $73 \pm 28$ & NS
\end{tabular}

ERP - effective refractory period, FP - fast pathway, SP - slow pathway, AV - atrioventricular, WCL - Wenckebach cycle length, TCL - tachycardia cycle length, $V A$ - ventriculoatrial interval, ms - milliseconds, NS - not significant; " patients showing discontinue AV conduction curve

Table III. Radiofrequency ablation and long-term follow-up results of groups

\begin{tabular}{lccc} 
Parameter & Group $1(n=16)$ & Group 2 $(n=266)$ & Value of $p$ \\
\hline Success rate, $n(\%)$ & $16(100)$ & $266(100)$ & NS \\
\hline $\begin{array}{l}\text { Successful ablation site, } n(\%): \\
\text { Midseptal }\end{array}$ & $9(56)$ & $155(58)$ & NS \\
\hline Posteroseptal & $7(44)$ & $111(42)$ & NS \\
\hline After successful ablation [ms]: & & & NS \\
\hline Maximum AH interval & $172 \pm 21^{*}$ & $166 \pm 35^{*}$ & NS \\
\hline AV node antegrade ERP & $304 \pm 31^{*}$ & $301 \pm 31^{*}$ & NS \\
\hline AV node WCL & $366 \pm 26^{*}$ & $356 \pm 35^{*}$ & NS \\
\hline PR prolongation, $n(\%)$ & 0 & $2(<1)$ & NS \\
\hline Transient AVB, $n(\%)$ & 0 & $1(<1)$ & NS \\
\hline Permanent complete AVB, $n(\%)$ & 0 & $0^{*}$ & NS \\
\hline Residual dual pathway, $n(\%)$ & $3(19)$ & $45(17)$ & NS \\
\hline Residual single echo beat, $n(\%)$ & $2(13)$ & $30(11)$ & NS \\
\hline Recurrence of AVNRT, $n(\%)$ & 0 & $2(<1)$ & NS
\end{tabular}

ERP - effective refractory period, AV - atrioventricular node, AVB - high-grade atrioventricular block, WCL - Wenckebach cycle length, * $p<0.001$ (as compared to preablation); \#one patient who received permanent pacemaker 1 year after the ablation procedure was not included in the statistical analysis. See text for detail information: $m s$ - milliseconds, NS - not significant

During follow-up, there was no need for permanent pacemaker implantation in any patient. One patient in group 2, a 65-year-old female, received a permanent pacemaker 1 year after the ablation because of symptomatic intermittent complete AV block. As we were not convinced that the ablation done a year ago was solely responsible for the AV block, this situation was not included in the statistical analysis.

\section{Follow-up after ablation}

The mean follow-up duration was $29 \pm 7$ (7 to 50) months. Recurrence of AVNRT was documented in only 2 patients in group $2(<1 \%)$ and these were successfully treated in a second session. The recurrence rate of AVNRT did not differ between groups (Table III). All other patients were asymptomatic during the follow-up period. 


\section{Discussion}

The data observed in this study suggest that: 1) repeated AVNRT episodes during RF application are more likely to be encountered in patients experiencing frequent tachycardia episodes in daily life; 2) the echo zone lasts longer in patients having more frequent AVNRT attacks; and 3) slow pathway ablation or modification may be accomplished safely and effectively during ongoing tachycardia in special circumstances.

The AVNRT can almost always be initiated with an atrial extrastimulus that is blocked antegradely in the fast pathway, conducted down the slow pathway and retrogradely up the fast pathway. However, the antegrade conduction down the slow pathway should be slow enough, which is defined as the critical $\mathrm{AH}$ interval, to allow for recovery of the fast pathway to conduct retrogradely, otherwise the reentry cannot occur [7]. Another prerequisite to the inducibility of AVNRT is the echo zone. The echo zone is defined as the zone in which atrial extrastimuli induce atrial echoes with or without AVNRT [8]. The outer and inner limits of this zone are the longest and shortest A1-A2 stimuli followed by an atrial echo, respectively. Reentry is likely to be inducible with extrastimuli slightly shorter than the outer limit of the echo zone and noninducible below the inner limit of the echo zone. The broader the echo zone, the more persistence of the critical AH delay, which plays a key role in the inducibility of AVNRT. Persistence of the echo zone after ablation generally means persistence of the critical delay of the $\mathrm{AH}$ interval. In our study, the echo zone was broader in group 1 , which could conceivably contribute to formation of the reentry circuit, making the tachycardia easier to induce and more difficult to control during ablation. Also, it could be one of the causes of the increased frequency of AVNRT episodes observed in this patient subset. On the other hand, premature depolarisations are known to be important for the initiation of AVNRT, while sympathetic predominance helps sustain the tachycardia. $\mathrm{Ni}$ gro et al. have shown that sympathetic predominance is responsible for sustained AVNRT episodes [9]. Although not statistically significant, higher incidence of structural heart disease and hypertension in group 1 may contribute to the development of frequent AVNRT attacks via increased premature beats and sympathetic predominance in these patients.

The principal debate of slow pathway ablation during ongoing tachycardia is the possibility of increased risk of AV block with this approach. During slow pathway ablation, only the development of a fast junctional tachycardia with a cycle length less than 350 ms is known to predict AV conduction block, and the risk is especially pronounced with cycle lengths under 300 ms [10]. However, in our experience and in the literature, the AVNRT cycle length is usually above 300 ms $[11,12]$. In our series, the cycle length of the AVNRT was above $300 \mathrm{~ms}$ in
$88 \%$ of the patients ( $356 \pm 44 \mathrm{~ms}$, range: $270-430 \mathrm{~ms}$ ) undergoing ablation during ongoing tachycardia. It can be speculated that ablation during ongoing tachycardia would not obscure the development of fast junctional rhythm in most of these patients, especially those with a cycle length under 300 ms, which is ominous for impending AV block.

While certain types of supraventricular tachycardia, such as focal atrial tachycardia or atrioventricular reentrant tachycardia, can be ablated during ongoing tachycardia after detailed mapping, slow pathway ablation or modification to eliminate AVNRT is usually done during sinus rhythm after the diagnosis is confirmed. The main motive for this approach is that the earliest endocardial activation point to be identified with mapping during ongoing AVNRT will not be the ablation point, unless the tachycardia is atypical fast-slow AVNRT. During typical AVNRT, the earliest endocardial activation during tachycardia is in the region of fast pathway, which is not the targeted ablation point. Other possible reasons could be the critical importance of this region for development of AV block and the difficulty in maintaining stable catheter position at the targeted slow pathway region during ongoing tachycardia.

It would be logical to determine the ablation point by mapping the earliest activation in the region of the slow pathway during atypical fast-slow AVNRT. However, as the general approach for ablation of any subtype of AVNRT is to ablate the presumed location of the slow pathway as determined predominantly electroanatomically, we apply the energy to the posteroseptal region that we think is the most distant from the AV node and the safest point, instead of trying to find the earliest activation at the posteroseptal region.

On rare occasions, AVNRT attacks cannot be controlled during EP study, making it necessary to proceed with ablation during ongoing tachycardia [2-4]. Successful ablation sites are usually located within $5 \mathrm{~mm}$ either above or below the level of the upper margin of the coronary sinus ostium [13]. This region is traditionally scanned during sinus rhythm, and RF energy is applied to sites where fractionated low-amplitude $A$ waves are recorded and the A/V ratio is below 0.5. However, atrial and ventricular activations are not readily discerned during typical AVNRT as they are activated almost simultaneously. For this reason, the anatomical approach has been favoured in this setting and sites with larger $V$ waves targeted for ablation, assuming that relative $A$ waves are not large. These sites were usually the posterior and sometimes middle third of the triangle of Koch, anterior to the coronary sinus, just superior to the tricuspid annulus insertion into the inferior interatrial septum. In particular the coronary sinus ostium was considered an important landmark, and energy applications were carried out in the $40^{\circ}$ left anterior oblique projection after confirmation of the final catheter tip position relative to 
the tricuspid annulus in the right anterior oblique projection.

In the last decade, cryoablation has evolved as an alternative technique to conventional RF catheter ablation [14]. Several clinical trials have shown the safety profile of this ablation technique, with not even a single permanent heart block reported until now for AVNRT [15]. As progressive ice formation at the catheter tip causes adherence to the adjacent tissue, ablation can also be safely performed during unstable catheter positions, or even during tachycardia, eliminating the risk of catheter dislodgement with termination of the tachycardia. Therefore, cryothermy could be especially useful in ablation of tachyarrhythmias in Koch's triangle at the expense of a relatively higher recurrence rate, which is the most encountered undesirable feature of this source of energy [16-18]. Due to its safety advantage, cryothermy is likely to be the preferred method of ablation of slow pathway during ongoing AVNRT in the near future.

The site of atrial stimulation may affect the inducibility of the tachycardia, probably because of different atrial inputs to the AV node or different functional refractory periods of the atria [19]. For this reason, the echo zone and tachycardia inducibility could have been different if induction studies were to be routinely repeated through the left atrium. However, our main purpose was not to examine the tachycardia inducibility and echo zones. Besides, the same stimulation protocol was carried out through the same atrial localisation in all patients. Thus, it can be assumed that the site of stimulation would not influence the differences in EP properties observed between the groups.

Cardiac autonomic tone is also influential on the EP behaviours of the AV node as well as inducibility of the tachycardia. Cardiac autonomic tone can be altered by pharmacologic stimulants, sedative medications, or blood pressure changes caused by rapid incremental pacing $[19,20]$. However, the EP data were obtained before administration of any pharmacological stimulants or sedatives.

The study may be criticised because of the relatively small sample size in the study group. However, this situation is not frequently encountered. In our study, 282 AVNRT ablations were evaluated, and we think the data is sufficient to derive a general clinical idea. Our aim is to provide a clinic sense rather than to show a statistical power to rule out $\mathrm{AV}$ block with this method or to say it is safe. It is also important to point out that the method should not be interpreted as a new strategy to treat all patients with AVNRT.

\section{Conclusions}

As the target sites for slow pathway ablation or modification are critical for risk of AV block, electrophysiologists prefer to work under sinus rhythm whenever pos- sible. However, our data demonstrate that RF catheter ablation or modification of the slow pathway during ongoing AVNRT may be achieved with acceptable shortand long-term efficacy and safety. Nonetheless, this approach needs to be clarified with large-scale studies.

\section{Acknowledgments}

The authors thank Satılmış Bakir and Ufuk Ecer for their valuable assistance.

\section{References}

1. Elvas L, Gursoy S, Brugada J, et al. Atrioventricular nodal reentrant tachycardia: a review. Can J Cardiol 1994; 10: 342-8.

2. Furushima $H$, Chinushi $M$, Sugiura $H$, et al. Radiofrequency catheter ablation for incessant atrioventricular nodal reentrant tachycardia normalized $\mathrm{H}-\mathrm{V}$ block associated with tachycardia-induced cardiomyopathy. J Electrocardiol 2004; 37: 315-9.

3. Selvaraj R, Arunprasath P, Karthikeyan B, et al. Uncommon presentation of a common tachycardia. Indian Pacing Electrophysiol J 2010; 10: 426-8.

4. Selvaraj R, Ananthakrishnapillai A, Sadasivam R, et al. "Pseudo PJRT"-fast-slow AV nodal reentrant tachycardia presenting with tachycardia-induced cardiomyopathy. Pacing Clin Electrophysiol 2013; 36: e4-6.

5. Otomo K, Suyama K, Okamura H, et al. Implications of 2:1 atrioventricular block during typical atrioventricular nodal reentrant tachycardia. J Interv Card Electrophysiol 2007; 19: 109-19.

6. Leitch J, Klein GJ, Yee R, et al. Invasive electrophysiologic evaluation of patients with supraventricular tachycardia. Cardiol Clin 1990; 8: 465-77.

7. Denes P, Wu D, Amat-y-Leon F, et al. The determinants of atrioventricular nodal re-entrance with premature atrial stimulation in patients with dual A-V nodal pathways. Circulation 1977; 56: 253-9.

8. Pintarić H, Zeljković I, Babić Z, et al. Electrophysiological predictors of propafenone efficacy in prevention of atrioventricular nodal re-entrant and atrioventricular re-entrant tachycardia. Croat Med J 2012; 53: 605-11.

9. Nigro G, Russo V, de Chiara A, et al. Autonomic nervous system modulation before the onset of sustained atrioventricular nodal reentry tachycardia. Ann Noninvasive Electrocardiol 2010; 15: 49-55.

10. Lipscomb KJ, Zaidi AM, Fitzpatrick AP, et al. Slow pathway modification for atrioventricular node re-entrant tachycardia: fast junctional tachycardia predicts adverse prognosis. Heart 2001; 85: 44-7.

11. Feldman A, Voskoboinik A, Kumar S, et al. Predictors of acute and long-term success of slow pathway ablation for atrioventricular nodal reentrant tachycardia: a single center series of 1,419 consecutive patients. Pacing Clin Electrophysiol 2011; 34: 927-33.

12. Amasyali B, Kose S, Aytemir K, et al. Atrioventricular nodal reentrant tachycardia with paroxysmal atrial fibrillation: clinical and electrophysiological features and predictors of atrial fibrillation recurrence following elimination of atrioventricular nodal reentrant tachycardia. J Interv Card Electrophysiol 2005; 13 : 195-201.

13. Yamane T, lesaka Y, Goya M, et al. Optimal target site for slow AV nodal pathway ablation: possibility of predetermined focal map- 
ping approach using anatomic reference in the Koch's triangle. J Cardiovasc Electrophysiol 1999; 10: 529-37.

14. Rodriguez-Entem FJ, Expósito V, Gonzalez-Enriquez S, et al. Cryoablation versus radiofrequency ablation for the treatment of atrioventricular nodal reentrant tachycardia: results of a prospective randomized study. J Interv Card Electrophysiol 2013; 36: 41-5.

15. deSisti A, Tonet J. Cryoablation of atrioventricular nodal reentrant tachycardia: a clinical review. Pacing Clin Electrophysiol 2012; 35: 233-40.

16. Kimman GP, Jordaens LJ. Transvenous radiofrequency catheter ablation of atrioventricular nodal reentrant tachycardia and its pitfalls: a rationale for cryoablation? Int J Cardiol 2006; 108: 6-11.

17. Skanes AC, Dubuc M, Klein GJ, et al. Cryothermal ablation of the slow pathway for the elimination of atrioventricular nodal reentrant tachycardia. Circulation 2000; 102: 2856-60.

18. Deisenhofer I, Zrenner B, Yin YH, et al. Cryoablation versus radiofrequency energy for the ablation of atrioventricular nodal re-entrant tachycardia (the CYRANO Study): results from a large multicenter prospective randomized trial. Circulation 2010; 122: 2239-45.

19. Josephson ME. Supraventricular tachycardias. In: Clinical cardiac electrophysiology. Josephson ME (ed.). Lippincott Williams \& Wilkins, Philadelphia 2008; 175-284.

20. Strickberger SA, Daoud EG, Niebauer MJ, et al. The mechanisms responsible for lack of reproducible induction of atrioventricular nodal reentrant tachycardia. J Cardiovasc Electrophysiol 1996; 7: 494-502. 